\title{
Simulations numériques de la dynamique des liquides et des verres
}

\author{
W. Kob
}

Laboratoire des Verres, Université de Montpellier 2, France

Introduction

- Pourquoi faire des simulations sur l'ordinateur?

- Quel genre de simulation est porteur a l'heure actuelle?

- La simulation en pratique?

- L'importance d'un bon potentiel.

- Dépendance des résultats en fonction de l'histoire de l'échantillon dans le cas des verres.

- Effets de taille finie

- L'importance de la mise en équilibre des systèmes

Simulation de $\mathrm{SiO}_{2}$

- Potentiel et détails de la simulation

- Dynamique: déplacement carré moyen, constante de diffusion, fonction de diffusion intermédiaire

- Propriété de factorisation

- Dépendance en q du facteur de Debye-Waller et relation avec la théorie du couplage des modes

Simulation de $\mathrm{Na}_{2} \mathrm{O}-x \mathrm{SiO}_{2}$

- Motivation

- Potentiel et détails de la simulation

- Constante de diffusion

- Distribution spatial des atomes de sodium: existence de canaux

- Facteurs de structure partiels

- Facteur de structure neutronique $\mathrm{S}_{\mathrm{n}}$ : comparaison de la théorie avec l'expérience

- Dynamique des atomes de sodium caractérisé par la partie propre (self) de la fonction de corrélation de van Hove

- Fonction de diffusion intermédiaire: cohérente et incohérente

- Temps de relaxation de la structure

- Dépendance en q de l'étirement

- résume 